\title{
TATOO: Testing and Analysis Tool for Object-Oriented Software
}

\author{
Amie L. Souter ${ }^{1}$, Tiffany M. Wong ${ }^{2}$, Stacey A. Shindo ${ }^{1}$, and Lori L. Pollock ${ }^{1}$ \\ ${ }^{1}$ Department of Computer and Information Sciences \\ University of Delaware, Newark, DE 19716 \\ \{souter, pollock, shindo\}@cis.udel.edu \\ 2 Department of Computer Science \\ Dartmouth College, Hanover, NH 03755 \\ Tiffany.M.Wong@dartmouth.edu
}

\begin{abstract}
Testing is a critical component of the software development process and is required to ensure the reliability, robustness and usability of software. Tools that systematically aid in the testing process are crucial to the development of reliable software. This paper describes a codebased testing and analysis tool for object-oriented software. TATOO provides a systematic approach to testing tailored towards object behavior, and particularly for class integration testing. The underlying program analysis subsystem exploits combined points-to and escape analysis developed for compiler optimization to address the software testing issues.
\end{abstract}

\section{Introduction}

Testing is a critical component of the software development process and is required to ensure the reliability, robustness and usability of software. Unfortunately, testing, and in particular ad hoc testing, is labor and resource intensive, accounting for $50 \%-60 \%$ of the total cost of software development 13 . Therefore, it is imperative that testing techniques be developed that provide as much automation and ease of use as possible. In particular, tools that systematically aid in the testing process are crucial to the development of reliable software.

Object-oriented features enable the programmer to design and develop software that is reusable and modular. Encapsulation, inheritance, and polymorphism are extremely useful to the programmer, but create difficulties for the tester. Encapsulation allows the programmer to create classes with state and functionality. All instantiated classes possess different state at different times through a program's execution, requiring many different test cases in order to adequately test the states of different objects. Complex class interactions are introduced through inheritance and class composition. These interactions also need to be tested, requiring test cases to exercise the complexities of these class relationships. Dynamic binding caused by polymorphism creates additional complexities when testing object-oriented software. A single polymorphic call site represents potential calls to one of a number of different methods with the same 
name. Each possible receiver type must be executed in order to adequately test a program.

In this paper, we present a Testing and Analysis Tool for Object-Oriented programs, TATOO, which embodies our novel code-based testing method focused on object manipulations [16]. Our tool establishes an environment to systematically test programs by providing: (1) automatically generated test tuples based on object manipulations, (2) test coverage information through code instrumentation and test coverage identification, (3) feedback about external influences that may affect the correctness of the testing runs, and (4) visualization of the underlying program representation that shows the interactions of objects in a program.

TATOO is composed of two subsystems: program analysis and testing. The program analysis subsystem's main objective is to produce the program representation needed for the testing subsystem. The testing subsystem generates the test tuples needed for generating test cases as well as provides test coverage information. In addition, the testing subsystem controls the user interface environment.

TATOO is a prototype tool implemented in Java, consisting of several components:

- The extended FLEX compiler infrastructure[11, which translates the source program into a program representation useful for testing.

- A test tuple generator that generates paths based on object manipulations.

- A Java graphical user interface that displays the source code and information corresponding to the component under test.

- The daVinci toolkit[8], which displays the program representation in a high quality manner, and facilitates communication with the Java GUI, allowing interaction between the graphical program representation and the source code and testing information.

- A code instrumentation tool.

- A trace analyzer, which provides coverage information about an executed program based on a given set of test tuples.

The major contribution of this work is a systematic framework for integration testing. The framework is based on a testing technique that focuses on object manipulations, and is capable of analyzing incomplete programs. Unlike previous work, the technique addresses the common situation of instance variables being objects and not primitive types.

The remainder of this paper is organized as follows. Section 2 provides background information on testing and object manipulations. An overview of TATOO is given in Section 3. Sections 4 and 5 describe the details of the TATOO subsystems. In Section 6, we evaluate the overhead attributed to the use of the program representation. Finally, we conclude with possible extensions to the tool. 


\section{Underlying Testing and Analysis Technique}

There are two main testing philosophies, namely, black-box and white-box testing. Black-box testing [2] does not use any knowledge of the internals of a program. The program is a black-box in which information about the source code is unknown; we only know what is provided from a program specification. Black-box tests are designed to uncover errors, but the focus of such testing is on verifying that a specified function operates according to the specification. Black-box testing has also been referred to as functional testing or specification-based testing.

White-box, structural, or code-based testing techniques are based on knowledge of the code. White-box testing is not an alternative to black-box testing; in fact, they complement each other and both should be performed. Test cases are derived through examining the program code and using well-defined data flow or control flow information about the program. Control flow-based techniques are motivated by the intuition that covering different control-flow paths would exercise a large proportion of program behaviors. For example, branch testing [7] is a control flow based testing method, which is based on exercising all the true and false outcomes of every branch statement. The general idea behind data flow testing [1410], is to generate test data based on the pattern of data used throughout a program.

Data flow testing is based on the premise that testing paths that read and write values stored into the same memory locations tests the behavior of a program in terms of its manipulation of data. More specifically, data flow testing is based on def-use pairs in a program, where a def of a variable is an assignment of a value to the variable via a read or assignment operation, and a use of a variable is a reference to the variable, either in a predicate or a computation. A def-use pair for variable $v$ is an ordered pair $(d, u)$ where $d$ is a statement in which $v$ is defined and $u$ is a statement that is reachable by some path from $d$, and $u$ uses $v$ or a memory location bound to $v$. Data flow testing uses def-use pairs in order to generate paths through the definition and use statements in the code. Then test data is generated based on those paths. The idea is that for each definition in the program, we want to exercise all of the uses of the definition.

Systematic testing techniques are categorized into different levels of testing. First, unit testing of object-oriented programs focuses on validating individual classes. As classes are combined or integrated together, integration testing is performed to validate that the classes function appropriately when combined together. Our research has focused on extending data flow testing to the objectoriented domain with special concern for testing instance variables that are objects and code-based integration testing of object-oriented components.

\subsection{Object Manipulation-Based Testing}

Our approach to code-based testing of object-oriented software seeks to provide coverage in terms of the elemental read and write actions, which is similar to data flow testing. We call our approach the $O M E N$ approach, because it is based on 
covering basic Object Manipulations in addition to using Escape iNformation to provide helpful feedback to the tester in an interactive testing tool environment 1

Object-oriented programming focuses on the data to be manipulated rather than the procedures that do the manipulating. An object-oriented program achieves its goals by creating objects of specific classes. The state of an object is encapsulated as a copy of all of the fields of data that are defined in the corresponding class definition. Actions are performed on an object by invoking methods defined in the class definition, often called sending a message to the object. A method invocation can modify and/or read the data stored in the particular object.

Table 1. Basic object manipulations.

\begin{tabular}{|l|l|}
\hline Object-related Statements & Object Manipulations \\
\hline copy $\mathrm{r}_{1}=\mathrm{r}_{2}$ & $\begin{array}{l}\text { read of reference } \mathrm{r}_{2} \\
\text { write to reference } \mathrm{r}_{1}\end{array}$ \\
\hline load $\mathrm{r}_{1}=\mathrm{r}_{2} . \mathrm{f}$ & $\begin{array}{l}\text { read of reference } \mathrm{r}_{2} \\
\text { read of field } \mathrm{r}_{2} . \mathrm{f} \\
\text { write to reference } \mathrm{r}_{1}\end{array}$ \\
\hline store $\mathrm{r}_{1} . \mathrm{f}=\mathrm{r}_{2}$ & $\begin{array}{l}\text { read of reference } \mathrm{r}_{2} \\
\text { read of reference } \mathrm{r}_{1} \\
\text { write to field } \mathrm{r}_{1} . \mathrm{f}\end{array}$ \\
\hline global load $\mathrm{r}=$ cl.f & $\begin{array}{l}\text { read of class variable } \mathrm{f} \\
\text { write to reference } \mathrm{r}\end{array}$ \\
\hline global store cl.f $=\mathrm{r}$ & $\begin{array}{l}\text { read of reference } \mathrm{r} \\
\text { write class variable } \mathrm{cl.f}\end{array}$ \\
\hline return $\mathrm{r}$ & read of reference $\mathrm{r}$ \\
\hline $\begin{array}{l}\text { object creation } \\
\mathrm{r}=\text { new Object }(\ldots . .)\end{array}$ & $\begin{array}{l}\text { create a new object } \\
\text { write to reference } \mathrm{r} \\
\text { MOD and USE }\end{array}$ \\
\hline $\begin{array}{l}\text { method invocation } \\
\mathrm{r}=\operatorname{r} \mathrm{r}_{0} \cdot \operatorname{methodname}\left(\mathrm{r}_{1}, \ldots, \mathrm{r}_{n}\right)\end{array}$ & $\begin{array}{l}\text { write to reference } \mathrm{r} \\
\text { read of references } \mathrm{r}_{0}-\mathrm{r}_{n} \\
\text { MOD and USE of } \mathrm{r}_{0} \text { 's } \\
\text { fields }\end{array}$ \\
\hline
\end{tabular}

In order to better understand the possible behaviors of an object-oriented program in terms of object manipulations, we identify the most elemental object manipulation as either a read or write action. The actions that a particular statement or method performs on an object can be decomposed into a sequence of these elemental actions. Table 1 depicts the elemental object manipulations performed by each object-related statement. We assume that the program has been preprocessed such that all statements that perform object manipulations

${ }^{1}$ In addition, we view the test cases and the results of executing the test cases as an omen to predicting the behaviors of the executing program in the production environment. 
have been expressed in the form of these basic statements. Due to aliasing and polymorphism, we may have a set of objects potentially referenced by each reference, but for these descriptions, we use the singular form. However, our analysis addresses the potential for a set of objects being referenced.

We extrapolate the concept of data flow testing to the testing of elemental object manipulations by defining a (write, read) association of a given object's state, extending this association to include object creation points. From Table 1 we can see that the statement that reads an object field is the load statement, while the store statement writes to an object field. To ensure that we do not miss any viable (write, read) pairs, we assume that a given load/store statement may $\mathrm{read} /$ write the field of any object which the reference is potentially referencing at that program point. Because objects are instantiated at run-time through executable statements, we extend (write, read) pairs to triples of the form (write, read, object creation) to reflect the fact that a test case should cover the creation of the object before any writes or reads to that object.

\subsection{Using Escape Analysis Information}

Escape analysis is a relatively new technique used for optimizing object-oriented codes, particularly Java codes $17 / 6 / 34$. The analysis is used to determine which synchronization operations are unnecessary and could be eliminated, as well as for reducing the number of objects that are unnecessarily allocated on the heap when they could be allocated on the stack.

We use escape analysis for testing, in order to provide useful feedback to the tester. For example, when a program is being tested and errors are uncovered, the tester or developer needs to find the cause of the error, i.e., debug the code. Our key insight is that the escape information will provide useful feedback to the tester about possible problem areas, where objects interact with outside code, which may be causing inadvertent changes to an object.

\section{TATOO System Architecture}

TATOO provides a tester with an interactive testing environment to systematically test software using the OMEN testing technique. The prototype testing tool allows the tester to visualize a graphical representation of the program, which characterizes how objects interact with other objects. In addition, the testing tool provides the tester with both visual and report-based coverage information about the program under test. TATOO also provides information about how objects interact with unanalyzed portions of code, as well as where objects may potentially escape through method calls or return statements. This information is useful in determining potentially fault prone sections of code.

The tool architecture is composed of two main subcomponents, namely the program analysis subsystem and the testing subsystem, as shown in figure 1 . The program analysis subsystem performs the required analysis to obtain the annotated points-to escape (ape) graph program representation described in section 
4.1 In addition, a term representation used to view the ape graph, and an annotation table which maintains the required information necessary for the testing subsystem are generated. After program analysis is performed, the testing subcomponent computes test tuples for testing the program component under test. In addition, the testing subcomponent provides a graphical user interface environment that supports two primary features: test coverage identification and program representation visualization. The test coverage identifier provides an environment that lets the user execute the program and then visualize coverage information. Alternatively, the primary purpose of the program representation visualizer is to allow the user to visualize object interactions.

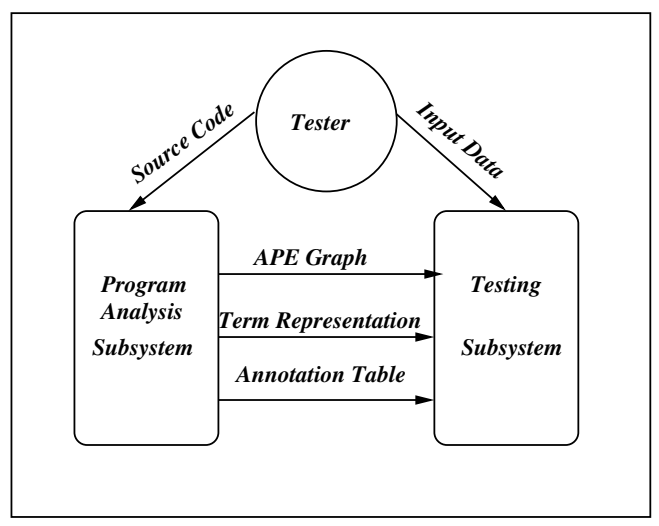

Fig. 1. Overall tool architecture.

\section{Program Analysis Subsystem}

Figure 2 depicts the subcomponents of the program analysis subsystem. Java bytecode produced from any Java source compiler is the input to the program analysis subsystem. The FLEX static analyzer [1] translates the Java byte code into an intermediate format based on object manipulations, and then performs points-to analysis to construct the $\boldsymbol{A}$ nnotated $\boldsymbol{P}$ oints-to $\boldsymbol{E}$ scape (ape) graph [16]. Annotations necessary for calculating read-write relationships between fields of objects are an important aspect of the ape graph.

The ape graph object, produced by the FLEX static analyzer, is then parsed to construct a graphical term representation needed to visually display the ape graph. In addition, a textual representation of the annotation table is generated, which consists of the annotations for each edge in the ape graph. The final output of the program analysis subsystem is the ape graph object, the ape graph graphical representation, and the annotation table. 


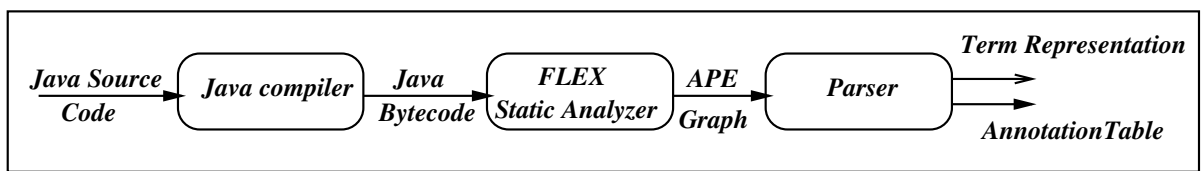

Fig. 2. Program analysis subsystem.

\subsection{Ape Graph Program Representation}

To develop the ape graph program representation, we extended and modified the points-to escape graph program representation [17] to exploit its ability to mimic object manipulations. The points-to escape graph representation combines points-to information about objects with information about which object creations and references occur within the current analysis region versus outside this program region. For our purposes, the current analysis region is the current component under test (CUT), where a component is not necessarily a class or method, but any grouping of methods. The points-to information characterizes how local variables and fields in objects refer to other objects. The escape information can be used to determine how objects allocated in one region of the program can escape and be accessed by another region of the program.

In the points-to escape graph, nodes represent objects that the program manipulates and edges represent references between objects. Each kind of object that can be manipulated by a program is represented by a different set of nodes in the points-to escape graph. There are two distinct kinds of nodes, namely, inside and outside nodes. An inside node represents an object creation site for objects created and reached by references created inside the current analysis region of the program. In contrast, an outside node represents objects created outside the current analysis region or accessed via references created outside the current analysis region. There are several different kinds of outside nodes, namely, parameter nodes, load nodes, and return nodes.

The distinction between inside and outside nodes is important because it is used to characterize nodes as either captured or escaped. A captured node corresponds to the fact that the object it represents has no interactions with unanalyzed regions of the program, and the edges in the graph completely characterize the points-to information between objects represented by these nodes. On the other hand, an escaped node represents the fact that the object escapes to unanalyzed portions of the program. An object can escape in several ways. A reference to the object was passed as a parameter to the current method, a reference to the object was written into a static class variable, a reference was passed as a parameter to an invoked method and there is no information about the invoked method, or the object is returned as the return value of the current method.

There are also two different kinds of edges. An inside edge represents references created inside the current analysis region. An outside edge represents references created outside the current analysis region. 
We have extended the points-to escape graph by adding annotations to edges in the graph. The annotations provide information about where basic object manipulations i.e., loads and stores of objects, occur within a program. Using the annotations, we are able to compute store-load i.e., (write-read) pairs for the objects in the program, which can be used in a manner similar to data flow testing.

For each method in the CUT, we build one ape graph per method. For each load/store of the reference represented by a particular edge $e$ in an ape graph, we maintain:

- a sequence of statement numbers, $\left(s_{1}, s_{2}, \ldots, s_{n}\right)$, where $s_{n}$ is the unique statement number of the load/store statement; $s_{1}, s_{2}, \ldots s_{n-1}$ contains the statement numbers of the call sites where this edge was merged into the caller's ape graph during interprocedural analysis performed during construction of the current method's ape graph. Statement $s_{1}$ is the statement number of the call site within the current analysis method which eventually leads to the load/store statement.

- a corresponding sequence of statement numbers, $\left(e v s_{1}, e v s_{2}, \ldots e v s_{n}\right)$, where each $e v s_{i}$ is the unique number of the earliest statement at which the statement $s_{i}$ could have an effect on other statements. We call this the earliest visible statement for $s_{i}, e v s_{i}$. The earliest visible statement $e v s_{i}=s_{i}$ when the statement $s_{i}$ is not inside a loop; otherwise $e v s_{i}=$ the statement number of the header of the outermost loop containing $s_{i}$.

\subsection{Example Annotation Construction}

Figure 3 shows an example set of annotations added to a single edge of an ape graph. The nodes in this graph represent objects created within the current analysis region; therefore, they are both inside nodes. The edge labeled top represents a reference from a field named top of the object of type Stack, annotated with both a load and store annotation. The annotations indicate that there exist both a load and store of the field top. Further, the location where the load and store occurs is maintained through the annotations. The annotation, (store 25-13-3), represents two calls, one invoked on line 25 of the program. The second call invoked on line 13 can lead to a store of an object into the field top at line 3. Similarly, the load of the field top occurs at line 7, following a chain of calls from lines 27 and 14. The above example does not include evs statement numbers, but they would be maintained in the same manner.

The annotation chains are easily constructed because the ape graphs for individual methods of the CUT are built during a reverse topological traversal over the call graph. Therefore, a callee's graph is always constructed before its callers' graphs. When constructing a caller's graph, the callees' annotations are simply merged into the caller's graph at the appropriate call site.

To emphasize the expressiveness of the ape graph, we include a complete ape graph for a simple method. Figure 4 illustrates the ape graph for method push. The black nodes represent outside nodes and the white nodes represent inside 


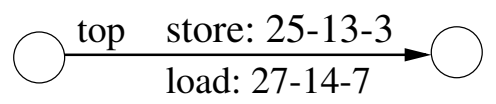

\section{Object of type Stack}

\section{Object of type Data}

Fig. 3. Illustration of ape graph annotation.

1: public push(Object e) \{

2: if $($ top $==$ null $)$

3: $\quad$ top $=$ new $\operatorname{Node}(e$, null);

4: else

5: $\quad$ top $=$ top.insert $(\mathrm{e}) ; \quad$ \}

6: Node (object e, Node n)\{

7: $\quad$ data $=\mathrm{e}$;

8: $\quad$ next $=\mathrm{n} ; \quad\}$

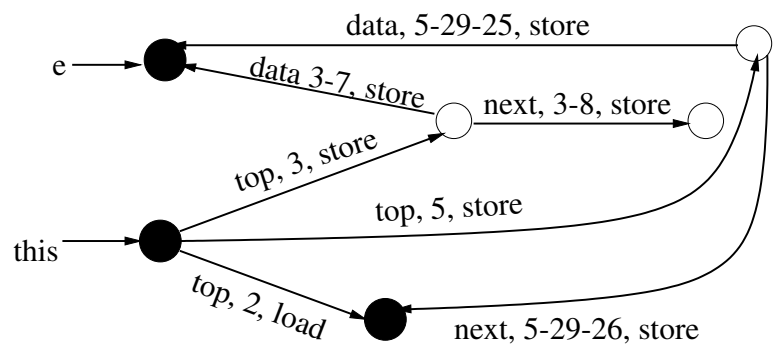

Fig. 4. Example of complete ape graph for a single method.

nodes. The graph was built by processing each statement in the method push. The constructor call to Node on line 3 maps the nodes from the Node graph into the ape graph for push, creating the edges (data, 3-7, store) and (next, 3-8, store). The annotations 3-7 and 3-8 indicate that a store occurred on lines 7 and 8 , through a call at line 3 . A similar mapping occurs at line 5, through the call to insert creating the other data and next edges. Due to space limitations, the code for the method insert does not appear.

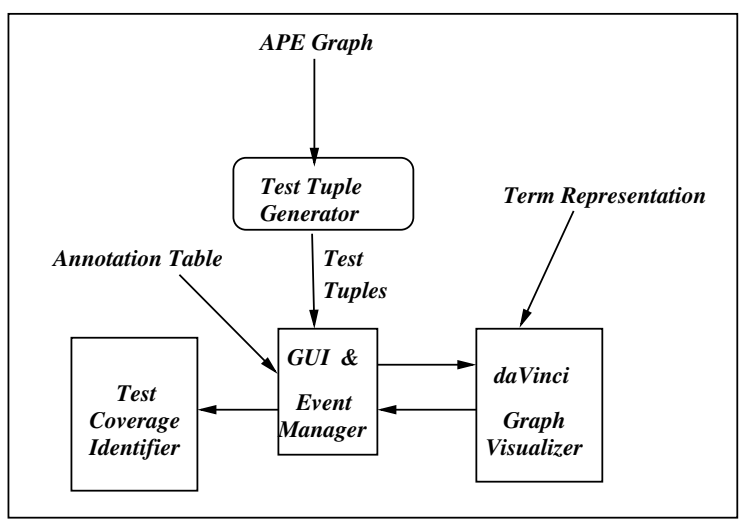

Fig. 5. Testing subsystem. 


\section{Testing Subsystem}

The testing subsystem, shown in figure 5, takes as input the ape graph object, the term representation necessary for graphically viewing the ape graph, and the annotation table, all generated from the program analysis subsystem. The following subsections describe the components of the testing subsystem, namely the test tuple generator and the test coverage identifier.

\subsection{Test Tuple Generator}

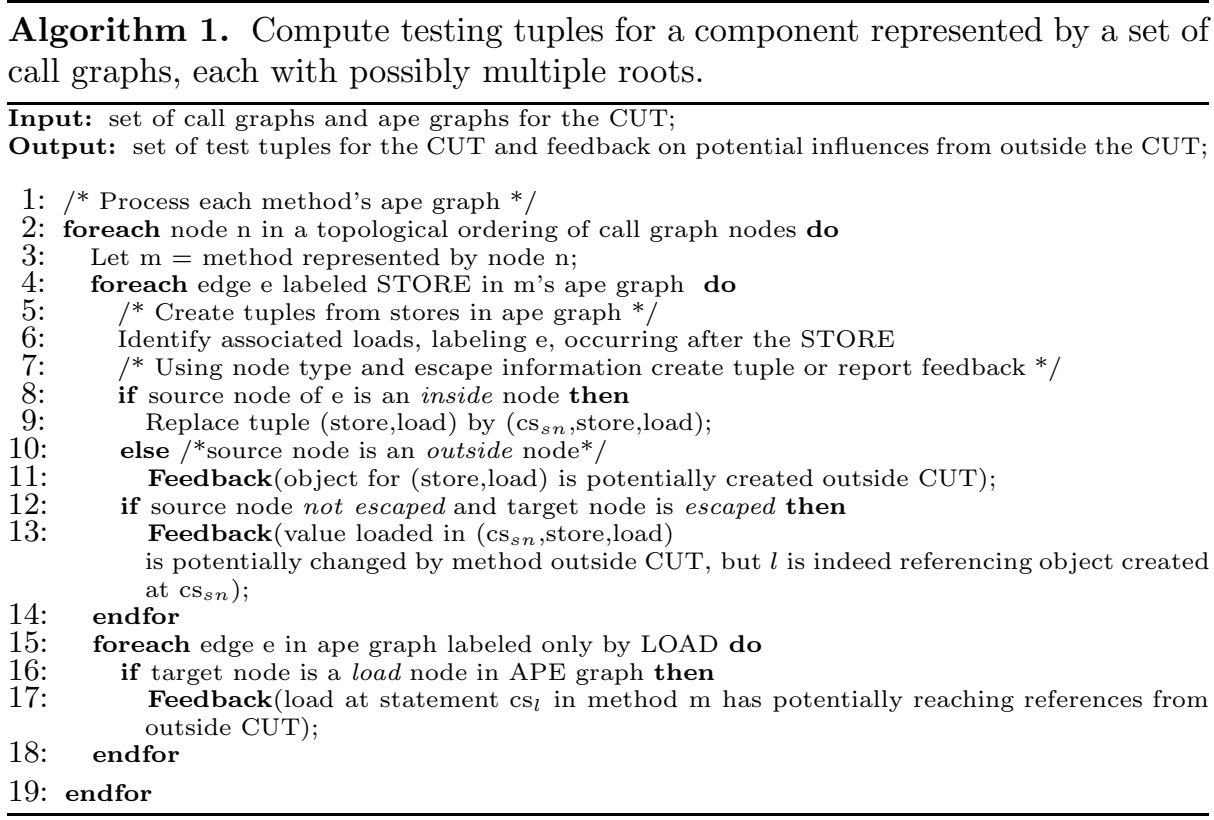

The test tuple construction algorithm, shown in Algorithm 1, computes a set of test tuples for the component under test(CUT), based on object manipulations. Starting at the root of each call graph of the CUT and proceeding in topological order, the method for each call graph node is processed once, by analyzing the node's ape graph. This processing order avoids creating duplicate tuples potentially identified due to subgraphs of invoked methods also appearing in a caller's ape graph. As a particular ape graph is analyzed, only unmarked edges (those not already processed in a caller's graph) are processed.

The algorithm processes each edge in a method's ape graph. For each annotation on an ape graph edge representing a store, the associated loads potentially occurring after the store are identified, and a (store,load) tuple is created. The annotations reflect the results of the flow sensitive points-to escape analysis used 
to build the ape graph. Thus, the evs and $c s$ statement numbers on these annotations are adequate to identify the reachable loads from a particular store. The object creation site associated with the (store,load) tuple is determined by the source node of the edge being analyzed. If the source node is an inside node, then the source node is the object creation site and the node number of the source node is used to complete the tuple for the (store,load) tuple. If the source node is an outside node, then the object is not created inside CUT, and feedback is given depending on the kind of the source node and whether it is interior or root of the call graph. Additionally, feedback is given when the target node of the ape graph edge being analyzed is escaped from CUT.

The algorithm also provides feedback for load nodes when a corresponding store is not present in CUT. This is represented by an ape graph edge that is labeled only with load annotations and no store annotations. The feedback provides the tester with information about the fact that an object creation site could have potentially occurred outside CUT, as well as the possibility that the load in CUT has potentially reaching references from outside CUT.

\subsection{Test Coverage Identifier}

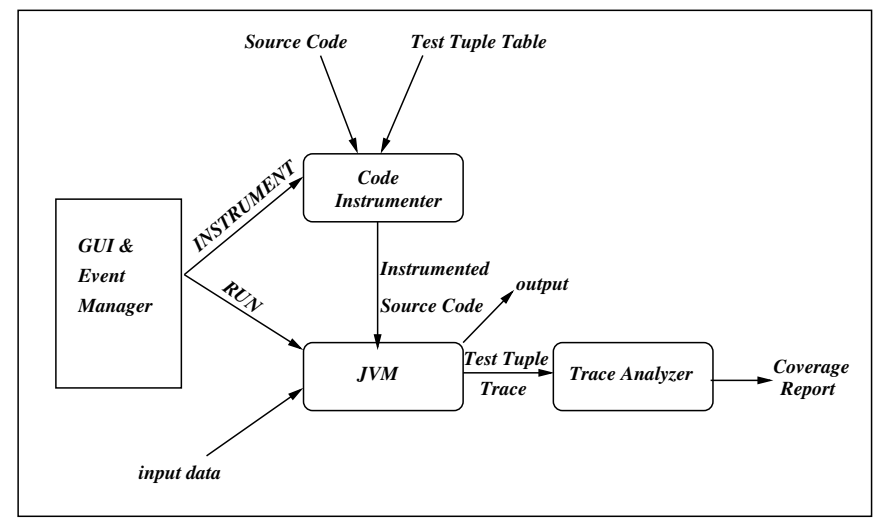

Fig. 6. Test Coverage Identifier.

The test coverage identifier provides an environment useful for providing test coverage information about the component under test. Figure 6 illustrates the subcomponents of the test coverage identifier. There are two user events, INSTRUMENT and RUN, which the event manager understands. An INSTRU$M E N T$ event invokes the code instrumenter, which takes the program source code and test tuple table as input. The source line numbers corresponding to the store-load-object creation site form the entries of the test tuple table, providing all the information necessary to instrument the source code. Simple print statements are inserted into the source code to produce instrumented code. The 
second event, $R U N$, invokes the component under test, prompting the user, if necessary, for input data, running the program on the JVM, and generating a test tuple trace file, in addition to the normal output of the program. The test tuple trace file is generated by running the instrumented source code, which provides information about the test tuples covered during the program execution.

Currently, we maintain coverage information for one run of the program. In the future, we will maintain information for multiple runs of the program, therefore providing more coverage information for the test suite of programs running on the component under test. The trace analyzer currently provides visual coverage information by analyzing the test tuple trace file and highlighting the source code and the corresponding test tuples that were covered during the program execution.

\subsection{Ape Graph and Test Tuple Visualizer}

The primary function of TATOOs ape graph and test tuple visualizer is for visualizing the ape graph representation of the CUT, which graphically displays object interactions. This subsystem is composed of the daVinci toolkit [8], a graphical user interface, and an event manager that communicates with daVinci, providing interaction between the source code, ape graph, annotations, and test tuples.

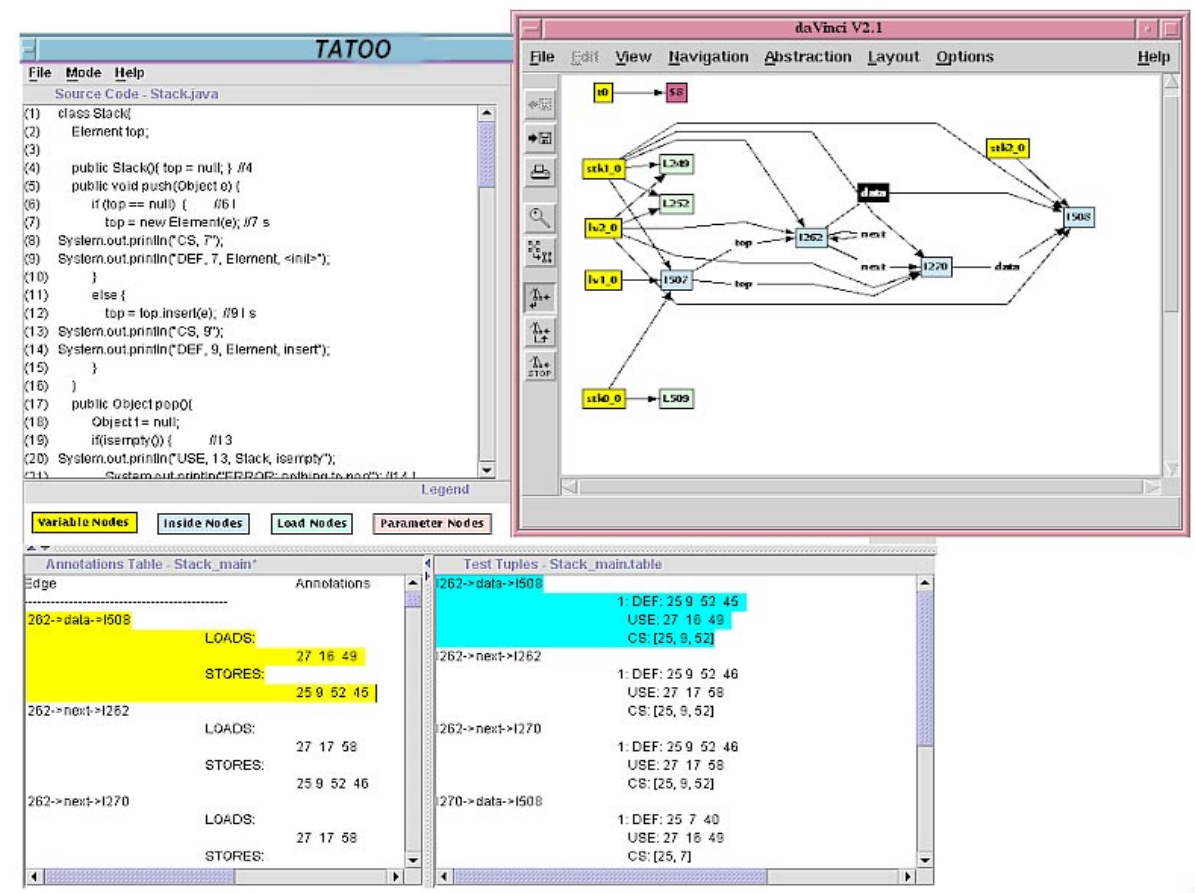

Fig. 7. The TATOO user interface including daVinci. 
Visualization of the ape graph is achieved through the daVinci graph drawing tool, which is an X-Window visualization tool for drawing high quality directed graphs automatically $[8]$. The term representation, produced by the program analysis subsystem, is the graphical representation used as input to daVinci. DaVinci not only displays the graph, but allows interaction between the graph and an external program. Our event manager communicates with daVinci through the API defined by the daVinci toolkit.

The graph visualizer interface of TATOO is shown in figure[7. A user can view the source code for a class, the annotations and test tuples produced for that class, and a graphical representation of the ape graph. In addition, the user is able to selectively view the ape graph and the source code, annotations, and test tuples corresponding to the selected graph segment. For example, a user can click on an edge in the daVinci graph window, and the source code, annotations, and test tuples corresponding to the selected edge are highlighted in their respective windows. The graph visualizer allows for a fine grain level of detail corresponding to how objects interact with other objects in a program. Its usefulness stems from the ability to statically visualize an object's fields and the points-to relationships constructed throughout the program. The escape information could also be visualized in a similar fashion, allowing for the user to click on the escape information, which would identify the region of code and the section of the graph that an object could escape through. The escape information is available to us, but this capability has not been included in our current prototype testing tool.

\section{Implementation and Evaluation}

TATOO has been implemented in Java and evaluated with a set of Java programs. Table 2 shows some general characteristics of the benchmark programs we have used with TATOO, as well as the storage requirements necessary for the ape graph. The characteristics include the number of lines of user code, the number of JVM instructions, the number of classes analyzed, and the number of methods analyzed. We have reported these numbers separately for user and library sizes in order to show that a relatively small program may rely heavily on libraries; therefore, analysis of the user program depends not only on the user code, but on the library code as well.

Table 2. Program characteristics and storage requirements.

\begin{tabular}{|l|l|r|r|r|r|r|r|r|r|r|r|r|}
\hline Name & Problem Domain & \# of & \multicolumn{2}{|c|}{ jvm instr } & \multicolumn{2}{|c|}{ classes } & \multicolumn{2}{|c|}{ methods } & \multicolumn{2}{|c|}{ ave size(Kb) } & \multicolumn{2}{|c|}{ max } \\
& & lines & User & Lib & User Lib & User Lib & User & Lib & size (Kb) \\
\hline compress & text compression & 910 & 2500 & 7070 & 17 & 90 & 50 & 301 & 6.1 & 1.1 & 43.0 \\
\hline db & database retrieval & 1026 & 2516 & 11648 & 9 & 100 & 240 & 306 & 14.5 & 0.9 & 98.6 \\
\hline mpeg & audio decompr & 3600 & 12019 & 7188 & 49 & 92 & 58 & 383 & 5.9 & 1.0 & 201 \\
\hline jlex & scanner generator & 7500 & 11000 & 7250 & 19 & 72 & 106 & 264 & 22.6 & 1.0 & 379 \\
\hline jess & expert system & 9734 & 15200 & 13005 & 108 & 105 & 468 & 436 & 13.9 & 1.1 & 668 \\
\hline
\end{tabular}


One concern in developing a code-based testing tool is the overhead of the underlying static analysis. We have experimentally evaluated the space overhead of our static analysis. The last three columns of table 2 show the average storage requirements of the ape graph per method (user and library), and the maximum ape graph storage requirement per benchmark. We computed the storage requirements by computing the sum of two products. The first product is the total number of nodes over all the ape graphs times the size of an ape graph node, and the second product is the total number of edges over all the ape graphs times the size of an edge. The storage requirement per ape graph is relatively small. The compositional nature of the ape graphs avoids the requirement of keeping all ape graphs in memory at once. The maximum ape graph represents the size needed to maintain the main method of the program. Essentially, the maximum ape graph contains the graphs from all of its callees, which were merged into itself.

The test tuple construction algorithm takes one pass over the call graphs representing the CUT. For each node in the call graph, it processes each unmarked edge of the ape graph for that method exactly once. The ape graph is easily extendible and the computation of additional test tuples can be performed in a demand-driven way as clients are added.

\section{Related Work}

Two proposed code-based testing tools based on data flow testing techniques include the Coupling Based Coverage Tool (CBCT) [1] and Orso's testing tool[12]. $\mathrm{CBCT}$ is a coverage-based testing tool that reports coverage metrics to the user, by instrumenting the source code. CBCT is based on a coupling-based testing technique, which is a data flow method based on coupling relationships that exist among variables across call sites, and is useful for integration testing. To our knowledge, CBCT has not been implemented. Orso's testing technique for integration testing is based on a data flow testing technique used for integration testing, in particular for polymorphic test coverage 12. His tool is similar to ours, but uses a different program representation, which does not provide testing feedback to the user. There is also no mention of how they deal with references or instance variables that are objects of different types in Java code.

Previous work on structural testing of object-oriented software has concentrated on data flow analysis for computing def-use associations for classes [9], testing of libraries in the presence of unknown alias relationships between parameters and unknown concrete types of parameters, dynamic dispatches, and exceptions [5], and developing a set of criteria for testing Java exception handling constructs 15 .

\section{Conclusions and Future Work}

We have presented TATOO, a testing and analysis tool for object-oriented software. The primary benefits of this tool are its ability to automatically generate 
code-based test tuples for testing, to determine test coverage through code instrumentation and test coverage notification, to provide feedback to the tester about external object interactions that could affect their result, and to visually display object interactions through the daVinci graph drawing tool.

In the future, we plan on extending this work in several ways. First, we plan on designing and implementing a new component to TATOO which automatically or semi-automatically generates test cases from the test tuples. Then, we plan to perform an experimental evaluation on different variations of the OMEN approach. Finally, we plan on designing and implementing a regression testing component for TATOO which will indicate portions of code that need to be re-tested after modifications have been made to the original code.

\section{References}

1. R. Alexander. Testing the polymorphic relationships of object-oriented components. Technical report, George Mason University, 1999.

2. B. Beizer. Software Testing Techniques. Van Nostrand Reinhold, 2nd edition, 1990.

3. B. Blanchet. Escape analysis for object oriented languages. Application to Java. In Proceedings of OOPSLA, November 1999.

4. J. Bodga and U. Hoelzle. Removing unnecessary synchronization in Java. In Proceedings of OOPSLA, November 1999.

5. R. Chatterjee and B. Ryder. Data-flow-based Testing of Object-Oriented Libraries. Technical Report 382, Rutgers University, March 1999.

6. J. Choi, M. Gupta, M. Serrano, V. Sreedhar, and S. Midkiff. Escape analysis for Java. In Proceedings of OOPSLA, November 1999.

7. T. Chusho. Test Data Selection and Quality Estimation Based on the Concept of Essential Branches for Path Testing. IEEE Transaction on Software Engineering, 13(5):509-517, May 1987.

8. M. Fröhlich and M. Werner. The Graph Visualization System daVinci. Technical report, Universität Bremen, Germany, September 1994.

9. M.J. Harrold and G. Rothermel. Performing Data Flow Testing on Classes. In Proceedings of the Symposium on the Foundations of Software Engineering, 1994.

10. M.J. Harrold and M.L. Soffa. Interprocedural Data Flow Testing. In Proceedings of the ACM Symposium on Testing, Analysis, and Verification, 1989.

11. M. Rinard et. al. FLEX. www.flex-compiler.lcs.mit.edu, 2000.

12. A. Orso. Integration Testing of Object-Oriented Software. PhD thesis, Politecnico Di Milano, 1999.

13. W. Perry. Effective Methods for Software Testing. John Wiley Inc, 1995.

14. S. Rapps and E. Weyuker. Selecting Software Test Data Using Data Flow Information. IEEE Transactions on Software Engineering, 11(4):367-375, April 1985.

15. S. Sinha and M.J. Harrold. Criteria for Testing Exception-Handling Constructs for Java Programs. In Proceedings on Software Maintenance, January 1999.

16. A. Souter and L. Pollock. OMEN: A Strategy for Testing Object-Oriented Software. In Proceedings of the International Symposium on Software Testing and Analysis, August 2000.

17. J. Whaley and M. Rinard. Compositional Pointer and Escape Analysis for Java Programs. In Proceedings of OOPSLA, November 1999. 